\title{
Comparison of cadmium-induced oxidative stress in Brassica juncea in soil and hydroponic cultures
}

Teresa Armas - Ana Paula Pinto - Amarilis de Varennes . Miguel Pedro Mourato - Luísa Louro Martins •

Maria Lurdes Simões Gonçalves - Ana Maria Mot:

Received: 19 March 2014/Accepted: 10 November 2014

(1) Springer International Publishing Switzerland 2014

\begin{abstract}
Aims The objective of this study was to investigate the response of Brassica juncea in the presence of $\mathrm{Cd}$, in hydroponic and soil experiments, and to conclude about common and divergent trends in both cultures.

Methods We studied the effect of $\mathrm{Cd}$ on growth, oxidative damage and antioxidant responses in roots and shoots of B. juncea grown in soil and hydroponic cultures, using typical time-scales for each one. Major ROS-scavenging enzymes such as catalase, ascorbate peroxidase and guaiacol peroxidase were evahuated, as well as lipid peroxidation.

Results Small Cd concentrations in the plant led to enhanced plant growth, while large $\mathrm{Cd}$ concentrations
\end{abstract}

impaired growth. The increase in lipid peroxidation observed in the presence of $\mathrm{Cd}$ was always greater in shoots than in roots. The physiological response to enhanced levels of reactive oxygen species in the presence of $\mathrm{Cd}$ included an increase in guaiacol peroxidase, ascorbate peroxidase and catalase activities, but those enzymes were not always enhanced in a similar manner in both cultures.

Canclusions The main factors responsible for the differences between the experiments in soil and nutrient solution, were the different $\mathrm{Cd}$ content in the plant tissues and the different time-scale of the experiments.

Keywords Antioxidant enzymes - Lipidperoxidation. Oxidative stress - Brassica juncea . Cadmium 